\title{
Telepathology by Realtime Screen Sharing Between Japan and Federated States of Micronesia: Opportunities and Challenges
}

\author{
Katsushige YAMASHIRO ${ }^{\mathrm{a}, 1}$ and Saimone VAMEAU ${ }^{\mathrm{b}}$ \\ ${ }^{a}$ Division of Diagnostic Pathology, Nikko Memorial Hospital, Japan \\ ${ }^{\mathrm{b}}$ Pathology Laboratory, Pohnpei State Hospital, Federated States of Micronesia
}

\begin{abstract}
We have been conducting pathological diagnosis of routine cases in the Federated States of Micronesia by telepathology since 2018, using realtime screen sharing via the Internet with Japan. The number of cases covered in the last three years was 378, and by specimen type, gynecologic specimens were the most common, followed by oral cavity / ENT, skin, and mammary gland. Thirty four out of 60 oral cavity cases were diagnosed as malignant including suspicious. The turnaround time between submission of the specimen and the report of pathological diagnosis was markedly decreased. For the continuous development of global telepathology, it is necessary not only to build a system but also to recruit or train those who perform it.
\end{abstract}

Keywords. Telepathology, realtime, service delivery

\section{Introduction}

The Federated States of Micronesia (FSM) is an island nation located north of the equator in the western south Pacific and consists of four states. A population of approximately 110,000 live centered around the main island of each state. The country faces a medical situation where they have no pathologists and because of this, in the past, pathology specimens were sent off island to the United States for diagnosis. To improve the situation, the authors have for the last three years performed the pathological diagnosis of routine cases using international telepathology connected by the Internet between Hokkaido, Japan and FSM. This paper introduces a summary of this work and provides some examples, followed by a discussion of some contemporary problems of the project.

\section{Background}

While working at the Hokkaido Cancer Center, the first author (KY) was asked if he would be interested in performing telepathology with someone from overseas. After expressing his interest, KY was introduced to the second author (SV) in FSM. SV is of Fijian nationality and is employed by the Pohnpei State Hospital as a clinical laboratory technician and has experience in preparing pathology specimens. In December 2017, KY traveled to Pohnpei to visit the hospital and pathology laboratory. He learned about the conditions regarding pathology diagnoses and was able to verify the specimen preparation techniques of SV. In February 2018, SV came to Japan and spent one month at the Hokkaido Cancer Center where he received training in the preparation of pathology

${ }^{1}$ Corresponding Author, Dr. K. Yamashiro, Shintomicho 1-5-13, Muroran City, Hokkaido, 051-8501 Japan; E-mail: sapporoyam@mac.com. 
specimens. A few months after this, diagnosis by telepathology was started.

\section{Methods}

The equipment items central to specimen preparation are as follows:

- Tissue Processor: STP 120 (Thermo Scientific),

- $\quad$ Embedding System: TES 99 MEDITE (MEDIZINTECHNIK),

- $\quad$ Microtome: HM 325 (Microm).

The staining and mounting of coverslip is performed manually: only Hematoxylin \& Eosin staining is possible. Macrophotography is performed to the maximum possible extent for specimens requiring cutting. The preparation of small specimens is left to the judgement of the responsible laboratory technician. The request for diagnosis should include the following clinical information:

- $\quad$ submission date,

- patient name, sex, age,

- physician's diagnosis,

- $\quad$ site, specimen type, objective, clinical findings, etc.

Once the glass slides have been prepared, an e-mail is sent from FSM to Japan, setting the time for screen sharing. The microscope used is an Elipse 50i (Nikon) with objective lenses of $2 x, 10 x$ and $40 x$. The microscope image is captured by digital camera BFLY-U323S6C-C (PointGrey) in real time and is projected to the computer screen using Panoptiq $^{\text {TM }}$ (ViewsIQ). The personal computer is an HP ProDesk (Intel Core i7-6700, 16GB RAM) using Windows 10 Pro with 32 inch-monitor (1920x1080 resolution).

The diagnosing pathologist in Japan uses a 2016 Apple Mac Book Pro 15-inch and screen sharing is performed with TeamViewer ${ }^{\mathrm{TM}}$. The diagnosis is performed by verbally designating the point of observation to the clinical laboratory technician who is operating the microscope and by requesting the lens magnification of the microscope. Once the diagnosis has been determined, it is entered into a text file on the computer in the FSM. Direct evaluation of the quality of the telepathology is very difficult. The authors actively participate in the Anatomical Pathology External Quality Assessment Programme of the Pacific Pathology Training Centre, New Zealand and strive to ensure the quality of their work.

\section{Results}

As of November 30, 2020, 378 cases have been diagnosed in almost three years (2018: 104 cases, 2019: 162 cases, 2020: 112 cases). This data has been summarized below in Table 1.

Patients ranged in age from 0 to 82 years, with an average age of 45.6 years. There were 124 males and 252 females, while 2 cases were unknown. The organs submitted, starting from the most abundant, are detailed in Table 1 and were as follows:

- gynecologic: 135 cases,

- oral and ENT: 60 cases,

- skin: 48 cases,

- mammary gland: 40 cases,

- gastrointestinal: 39 cases. 
Table 1. Number of cases by organ and results of pathological diagnosis

\begin{tabular}{|c|c|c|c|c|c|c|c|c|}
\hline \multirow[t]{6}{*}{ Gynecologic } & Total & 135 & GI tract \& related & Total & 39 & Urologic & Total & 7 \\
\hline & B & 101 & & B & 28 & & $\mathbf{B}$ & 4 \\
\hline & 1 & 4 & & $\mathbf{I}$ & 2 & & 1 & $\mathbf{0}$ \\
\hline & $\mathbf{s}$ & 3 & & $\mathbf{s}$ & $\mathbf{0}$ & & $\mathbf{s}$ & 1 \\
\hline & $\mathbf{M}$ & 27 & & M & 9 & & M & 2 \\
\hline & $\mathbf{I}+\mathbf{S}+\mathbf{M} /$ Total & 0.252 & & I $+\mathbf{S}+\mathbf{M} /$ Total & 0.285 & & I $+\mathbf{S}+\mathbf{M} /$ Total & 0.429 \\
\hline \multirow[t]{6}{*}{ Oral + ENT } & Total & 60 & Soft tissue & Total & 20 & Bone \& Joint & Total & 6 \\
\hline & B & 18 & & B & 16 & & B & 5 \\
\hline & $\mathbf{I}$ & 8 & & $\mathbf{I}$ & 1 & & $\mathbf{I}$ & $\mathbf{0}$ \\
\hline & $\mathbf{s}$ & 11 & & $\mathbf{s}$ & $\mathbf{0}$ & & $\mathbf{s}$ & $\mathbf{0}$ \\
\hline & M & 23 & & M & 3 & & $\mathbf{M}$ & 1 \\
\hline & $\mathbf{I}+\mathbf{S}+\mathbf{M} /$ Total & 0.7 & & I+S+M/Total & 0.2 & & $\mathbf{I}+\mathbf{S}+\mathbf{M} /$ Total & 0.167 \\
\hline \multirow[t]{6}{*}{ Skin } & Total & 48 & Lymph node & Total & 10 & Eye & Total & 3 \\
\hline & B & 33 & & B & 3 & & B & 3 \\
\hline & 1 & 2 & & $\mathbf{I}$ & 1 & & $\mathbf{I}$ & $\mathbf{0}$ \\
\hline & $\mathbf{s}$ & 2 & & $\mathbf{s}$ & 1 & & $\mathbf{s}$ & $\mathbf{0}$ \\
\hline & $\mathbf{M}$ & 11 & & $\mathbf{M}$ & 5 & & $\mathbf{M}$ & $\mathbf{0}$ \\
\hline & $\mathbf{I}+\mathbf{S}+\mathbf{M} /$ Total & 0.313 & & $\mathbf{I}+\mathbf{S}+\mathbf{M} /$ Total & 0.7 & & $\mathbf{I}+\mathbf{S}+\mathbf{M} /$ Total & $\mathbf{0}$ \\
\hline \multirow[t]{6}{*}{ Breast } & Total & 40 & Thyroid & Total & 7 & Salivary gland & Total & 1 \\
\hline & B & 23 & & B & 4 & & B & 1 \\
\hline & I & 1 & & $\mathbf{I}$ & 2 & & $\mathbf{I}$ & $\mathbf{0}$ \\
\hline & $\mathbf{s}$ & 2 & & $\mathbf{s}$ & $\mathbf{0}$ & & $\mathbf{s}$ & $\mathbf{0}$ \\
\hline & $\mathbf{M}$ & 14 & & $\mathbf{M}$ & 1 & & $\mathbf{M}$ & $\mathbf{0}$ \\
\hline & $\mathbf{I}+\mathbf{S}+\mathbf{M} /$ Total & 0.425 & & I+S+M/Total & 0.429 & & $\mathbf{I}+\mathbf{S}+\mathbf{M} /$ Total & o \\
\hline & & & & & & & & \\
\hline
\end{tabular}

Two cases where the specimen was too small and unsuitable for diagnosis, were excluded. The number of cases determined as malignant (M), suspected malignant (S), indeterminate (I) and benign (B) are also shown in Table 1. For each organ. 'Indeterminate' means that it was difficult to judge benign or malignant. Oral and ENT related specimens had the highest percentage of malignant, suspicious and indeterminate diagnoses. Of these, oral specimens of the buccal mucosa, etc. had highest frequency with 39 cases.

As reported by SV, the turnaround time between submission of the specimens and the report of pathological diagnosis was evaluated for the years 2019 and 2020 when glass slide preparation became routine. The turnaround time for cases requested by the Pohnpei State Hospital was four days, for the other states of Kosrae, Yap and Chuck, turnaround time was seven to eight days.

\section{Examples}

This section highlights some of the more interesting telehealth cases. Example 1 relates to oral cancer in a male in his $40 \mathrm{~s}$, a betel nut chewer. Photographs taken at Pohnpei State Hospital with a x10 and x40 objectives are shown in Figure 1 and 2. Screen shots from the monitor displayed through TeamViewer ${ }^{\mathrm{TM}}$ in Japan are shown in Figure 3 and 4 . The latter two may not be as clear as the display appeared under a microscope. It is well known that oral cancer frequently occurs in betel nut chewers.

Example 2 relates to Hansen's disease in a male in his 40s. A skin biopsy was performed because of suspected Hansen's disease, new to the author in 40 years of experience as a pathologist (Figure 5 and 6). 


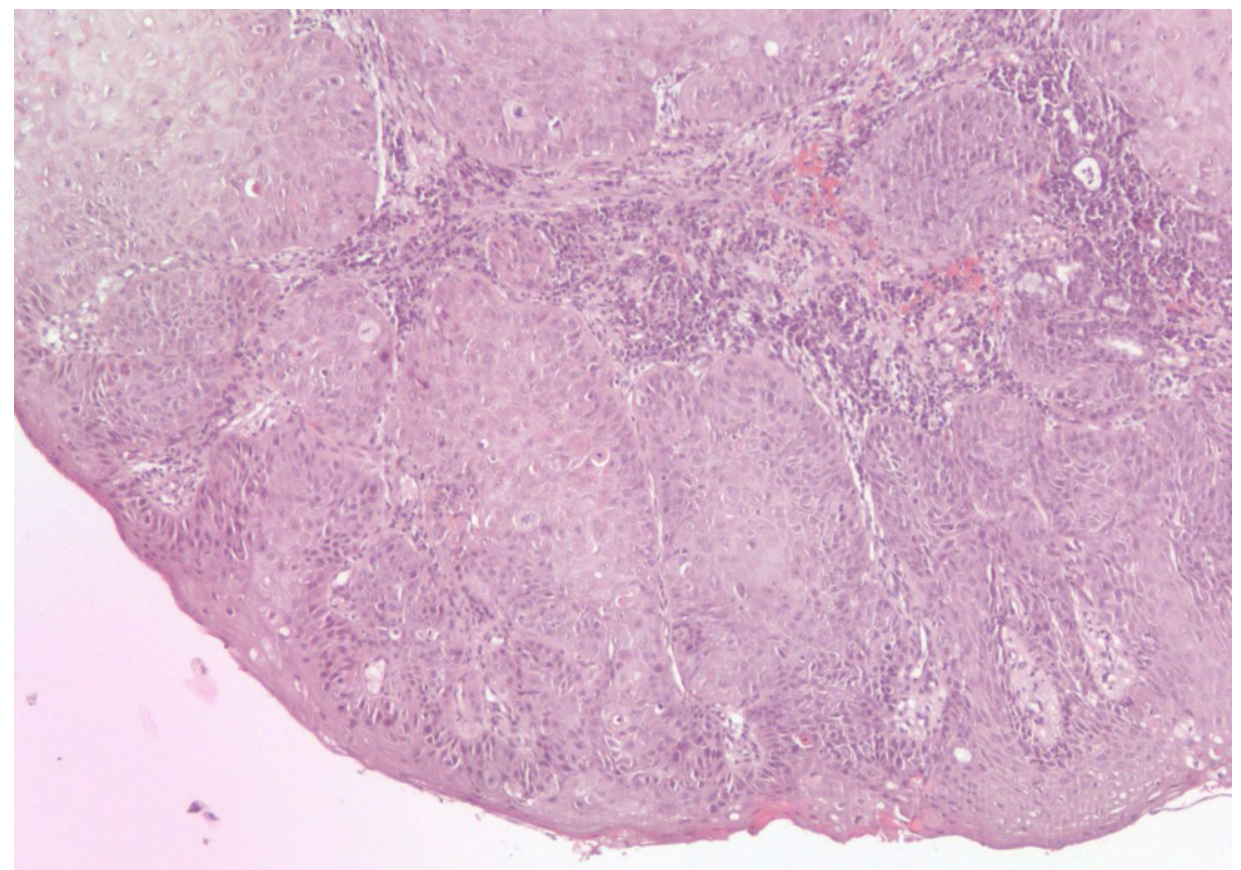

Figure 1. Squamous cell carcinoma, invasive, buccal mucosa. x10. Captured in FSM.

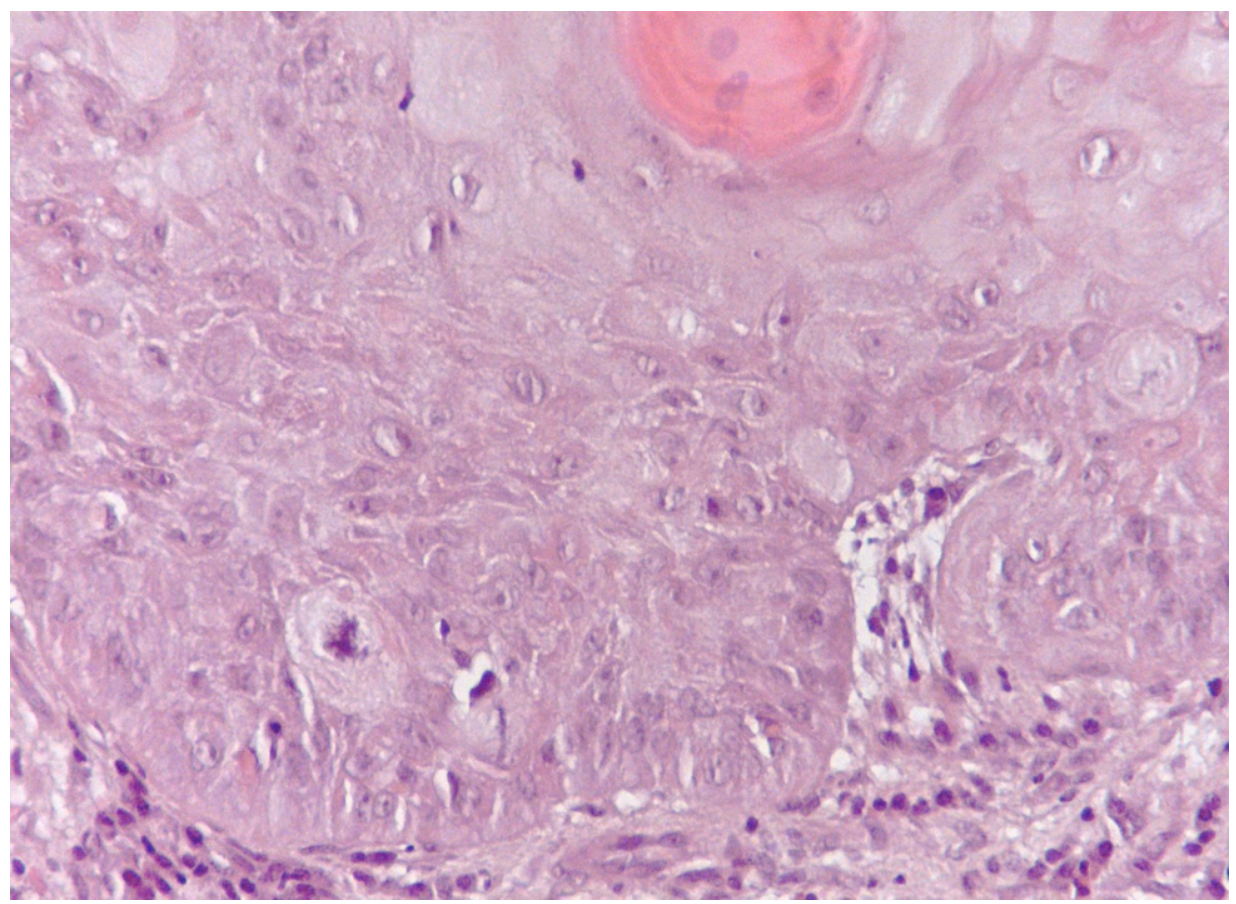

Figure 2. Squamous cell carcinoma, invasive, buccal mucosa. $x$ 40. Captured in FSM. 


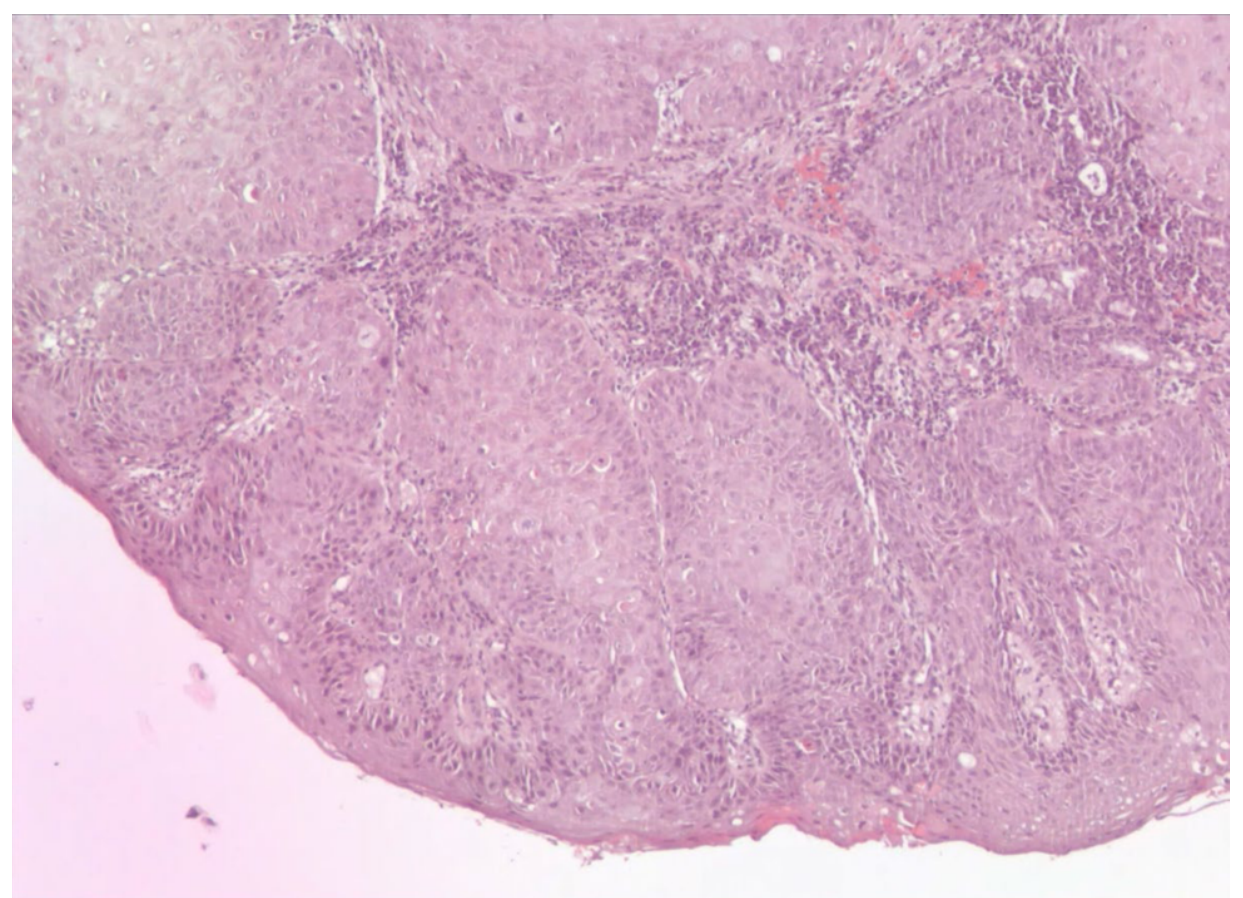

Figure 3. Realtime screen image transmitted by Teamviewer. Captured in Japan. Same image as Fig. 1.

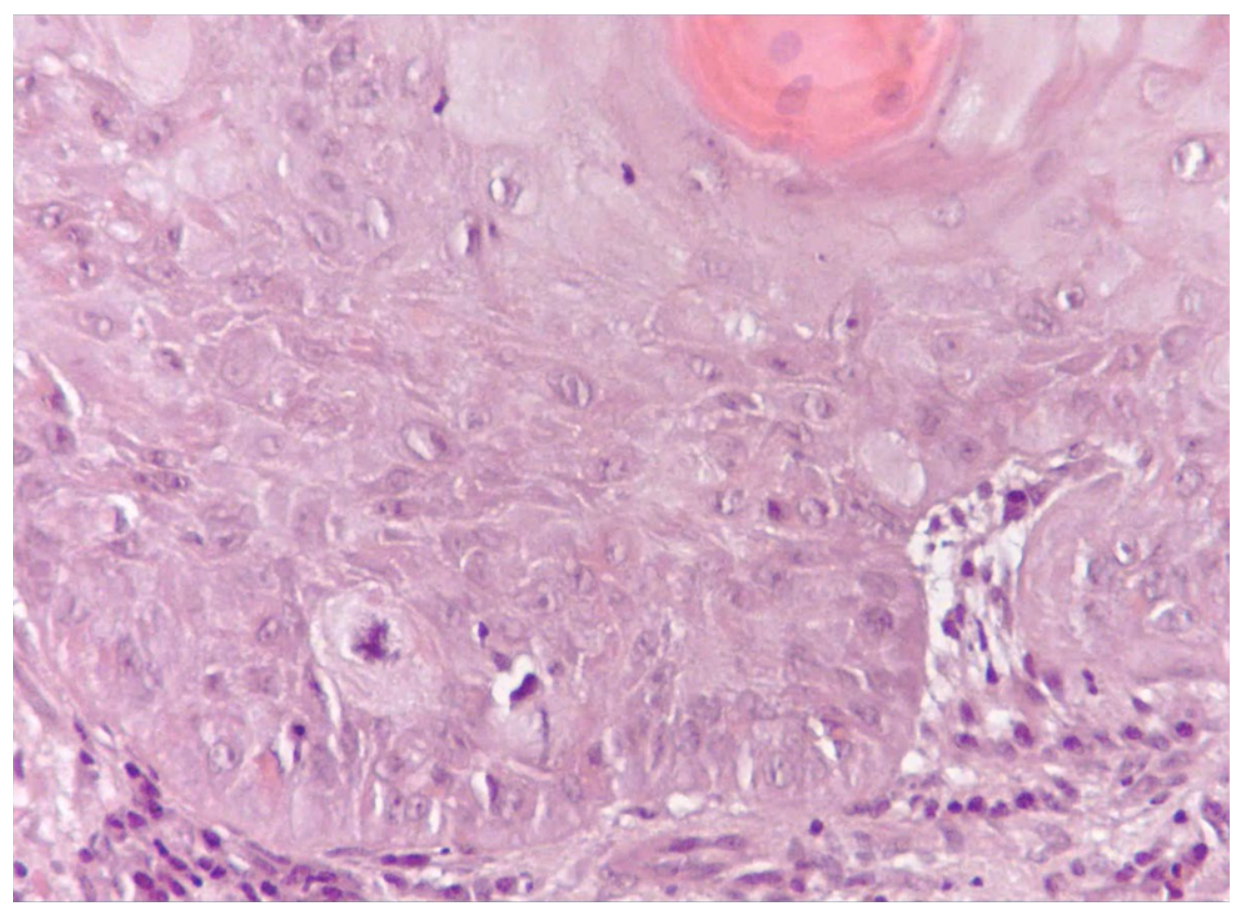

Figure 4. Realtime screen image transmitted by Teamviewer. Captured in Japan. Same image as Fig. 2. 


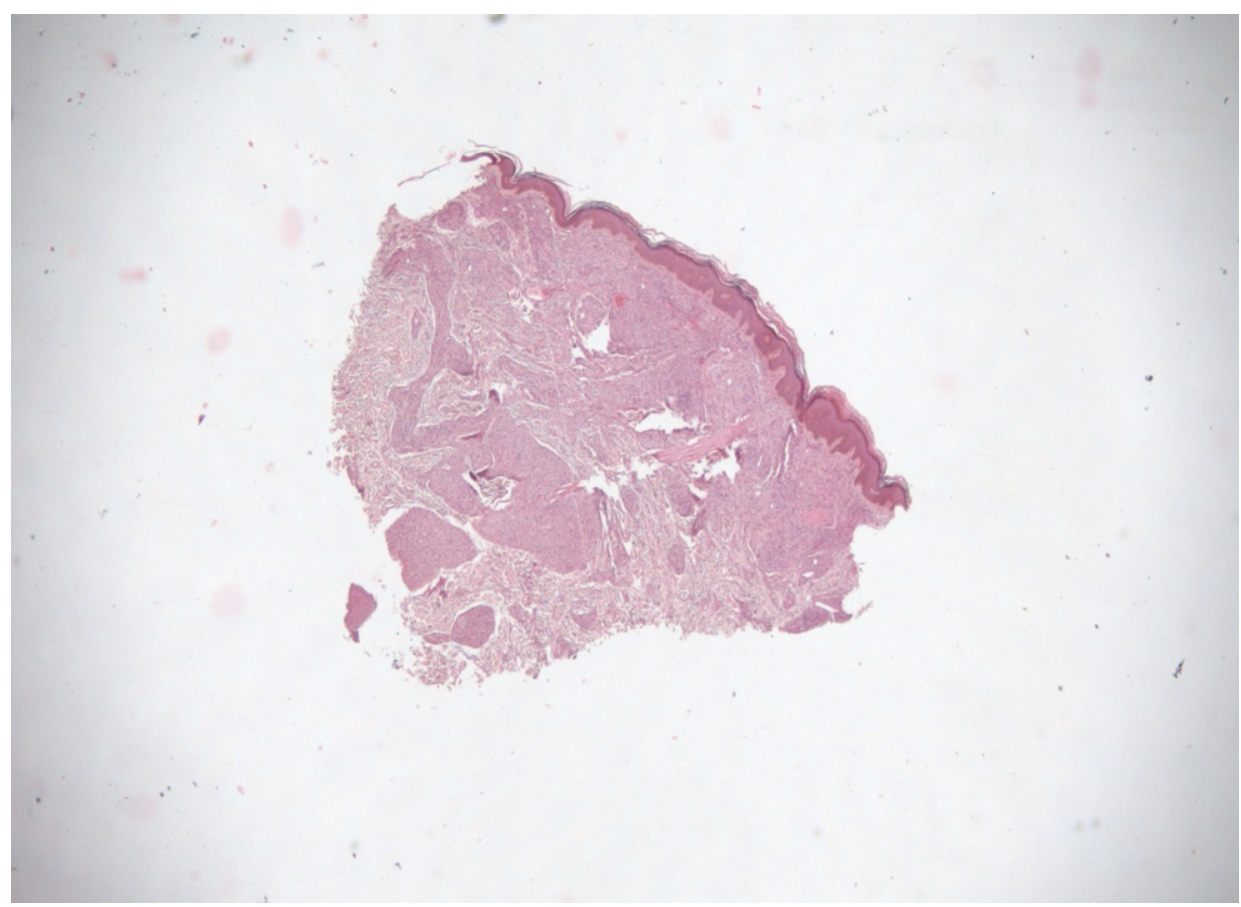

Figure 5. Lepromatous leprosy. Histiocytic aggregation in dermis with irregular margin, looks like cancer cell growth. $\mathrm{x} 2$.

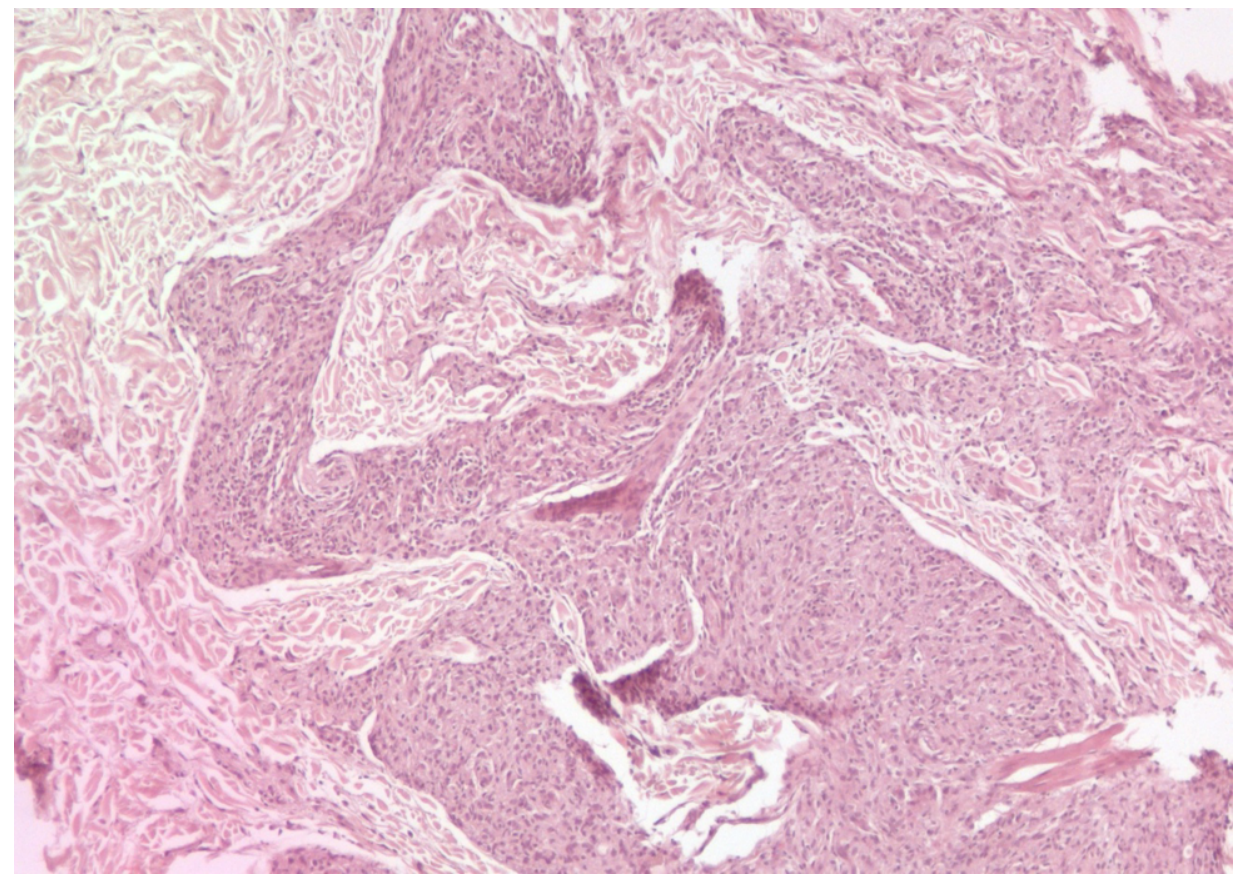

Figure 6. The lesion contains few lymphocytes x10. 


\section{Discussion}

Telepathology began to gain acceptance as a medical technology around 1990 and was one of the first fields to be realized in telemedicine [1]. At that time, it was first used for rapid diagnosis during surgery [2], however its application for the diagnosis of routine paraffin block specimens did not advance, and its spread into the field of cytology was limited $[3,4,5,6]$. On the other hand, telepathology device development has focused upon whole slide imaging/virtual slide (WSI/VS) and furthermore, the interest of pathologists seems to be moving towards artificial intelligence (AI) aided diagnosis using this system $[7,8]$. As the first 20 years of the 21 st century is coming to an end, some countries are still unable to receive the benefit of pathological diagnosis and the authors believe that those who have the means to solve that problem should come forward and do so. The FSM is an expansive oceanic federated nation. Consequently, with a vast area and sparse population, it can be imagined there would be difficulties in creating systems that any modern nation should have. The underlying medical system is representative of this. For example, with the exception of nurses, a system of education and training for medical staff has yet to be established in the FSM. They are reliant upon the supply of doctors from other countries. Pharmacists, radiologists, clinical laboratory technicians, etc. are in the same situation. Because of this, constructing a hospital with adequate human resources is challenging.

As an example, consider the treatment of cancer, for which there has been considerable advances in the accumulation of knowledge and organized treatment procedures. In order to perform a certain level of treatment unavailable in FSM requires the patient to be treated at a foreign facility. Without first establishing a diagnosis, the patient cannot be referred to such a facility. If a pathological specimen is sent to a foreign laboratory capable of providing a diagnosis, then a pathological diagnosis can be achieved. If it takes one to two months for the diagnosis, it may be too late for the patient. This type of time lag has previously occurred in the FSM. Telepathology is the tool that can change this situation. WSI/VS has developed well enough to handle this task and is considered a refined system. However, we did not adopt this system because we had no funding for this project. We made no demands upon the governments of the FSM nor Japan to consider this experimental telepathology. The Pohnpei State Hospital has an Internet connection, although it is quite inadequate, and the pathology laboratory microscope has a digital camera connected to a computer connected to the Internet.

We considered using the author's personal computer and private WIFI connection for telepathology. In fact, we have used the method of real-time screen sharing for telecytology in Japan for over a dozen years [3]. Although financial support was not available at the start, the authors are optimists and continued to establish this telepathology operation between Japan and FSM, with the hopes that the success of the project would attract financial support. There were no time zone problems in the connection between countries or regions for real-time screen sharing because the time difference between Japan and Pohnpei is only two hours. One hour of connection time is possible if telepathology is initiated at 14:00 Japan Standard Time, which will allow the clinical laboratory technician to finish work at the end of a typical workday. Excluding difficult cases, two to three cases with a normal degree of difficulty can be diagnosed in one hour. Real time screen sharing is not without its problems. Operation of the microscope is performed by the clinical laboratory technician of the FSM but because of the first author's weak English conversation skills, it has been difficult and we had to adapt. Furthermore, the transmitted microscope image compared to looking 
through the microscope is not as clear. However, this was not such a major concern because of the smooth transmission of the image. The laboratory technician made efforts to prepare clear glass slides after listening to the first author's occasional comments and has become proficient at operating the microscope.

There are a number of other logistical problems. Firstly, the first author is 68 years old and it may be difficult for him to continue this work for another 10 years. It is hoped that another pathologist supportive of these activities would emerge. International support subsidized by governments of multiple nations to create pathologists would be desirable and the author would be glad if this report could be an inspiration for this. Secondly, a current request to perform Pap test cytology has been made by the FSM. It is well known that cytology is the collaborative work of a pathologist and a cytotechnologist. The cytotechnologist is a specialist who not only prepares the glass slide but also performs screening and identification of the neoplastic cell, etc. and requires time and an education system to train them. There are no cytotechnologists in the FSM. In order to make this possible, the recruitment and training of a motivated clinical laboratory technician is necessary. Thirdly, SV is a clinical laboratory technician working from FSM with the first author, but he will, in time, also retire and require a successor. It can be imagined that a person having the knowledge and technical skills to prepare a pathology specimen will be in demand not only in the FSM but in clinical practice in the countries of greater Micronesia and oceanic nations south of the equator. These individual countries need to work together to create a system to train specialized medical technologists. International telepathology is not limited to the construction of a system but fundamentally it also requires a mechanism for local human resources development.

\section{Acknowledgements}

The authors would like to thank the FSM Government for its constant and sincere support in accomplishing the telepathology project and also thank Fredrick Miller, Medical Information Division of Nikko Memorial Hospital, Japan, for his assistance with the English proofreading, etc. in the preparation of this manuscript.

\section{References}

[1] Weinstein RS, et al. Prospects for telepathology. Hum Pathol 1986;17:433-434.

[2] Nordrum I, Engum B, Rinde E, et al. Remote frozen section service: A telepathology project in northern Norway. Hum Pathol.1991;22: 514-518.

[3] Pantanowitz L, Wiley CA, Demetris A, et al. Experience with multimodality telepathology at the University of Pittsburgh Medical Center. J Pathol Informatics. 2012;3;45

[4] Yamashiro K, Kawamura N, Matsubabyashi S, et al. Telecytology in Hokkaido Island, Japan: results of primary telecytodiagnosis of routine cases. Cytopathology 2004;15:221-227

[5] Bernard C, Chandrakanth SA, Cornell IS, et al. Guidelines from the Canadian Association of Pathologists for establishing a telepathology service for anatomic pathology using whole-slide imaging. J Pathol Informatics. 2014;5;15

[6] Tabata K, Mori I, Sasaki T, et al. Whole-slide imaging at primary pathological diagnosis: Validation of whole-slide imaging-based primary pathological diagnosis at twelve Japanese academic institutes. Pathol Int 2017; 67: 547-554

[7] Parwani AV. Next generation diagnostic pathology: use of digital pathology and artificial intelligence tools to augment a pathological diagnosis. Diagn Pathol 2019;14:138. Available: https://doi.org/10.1186/s13000-019-0921-2

[8] Chen M, Zhang B, Topatana W. et al. Classification and mutation prediction based on histopathology H\&E images in liver cancer using deep learning. npj Precis. Onc. 4, 14 (2020). https://doi.org/10.1038/s41698020-0120-3 